\title{
LE PROBLÈME PUBLIC DU HARCÈLEMENT DE RUE : DYNAMIQUES DE PUBLICISATION ET DE PÉNALISATION D’UNE CAUSE FÉMINISTE
}

\author{
Carole Gayet-Viaud, Mischa Dekker
}

Médecine \& Hygiène | «Déviance et Société »

2021/1 Vol. 45 | pages 7 à 23

ISSN 0378-7931

Article disponible en ligne à l'adresse :

https://www.cairn.info/revue-deviance-et-societe-2021-1-page-7.htm

Distribution électronique Cairn.info pour Médecine \& Hygiène.

(C) Médecine \& Hygiène. Tous droits réservés pour tous pays.

La reproduction ou représentation de cet article, notamment par photocopie, n'est autorisée que dans les limites des conditions générales d'utilisation du site ou, le cas échéant, des conditions générales de la licence souscrite par votre établissement. Toute autre reproduction ou représentation, en tout ou partie, sous quelque forme et de quelque manière que ce soit, est interdite sauf accord préalable et écrit de l'éditeur, en dehors des cas prévus par la législation en vigueur en France. Il est précisé que son stockage dans une base de données est également interdit. 


\title{
Le problème public du harcèlement de rue: dynamiques de publicisation et de pénalisation d'une cause féministe
}

\author{
Carole GAYET-VIAUD \\ CNRS-CESDIP \\ EHESS-CEMS
}

Mischa DEKKER

EHESS-LIER 
Les rapports entre hommes et femmes dans les espaces publics urbains font, depuis plusieurs décennies maintenant, l'objet d'un travail militant destiné à ériger en "problème public» (Cefaï, Terzi, 2012) certains comportements récurrents à l'égard des femmes relativement banalisés auparavant. D’abord développée outre-Atlantique (Kearl, 2010), la lutte contre ce qui est désormais décrit et dénoncé comme le phénomène du «harcèlement de rue» a pris son essor en Europe au tournant des années 2010, lors de la médiatisation inédite de certaines expériences féminines de la ville (Lieber, 2008; Peeters, 2012), relayées ensuite par la création de collectifs féministes militants, spécifiquement dédiés à ce problème ${ }^{[1]}$.

Des espaces d'engagement, à la fois civique et partisan, se sont ainsi graduellement constitués autour de la question, jusqu'à ce que des collectifs d'acteurs s'organisent et s'imposent comme porteurs de cause et «propriétaires» (Gusfield, 2009) du problème public du harcèlement de rue, diffusant et consolidant sa formulation, promouvant la lutte contre ce phénomène, selon des configurations diverses, à l'échelle internationale, et notamment au sein de l'espace européen. À la faveur de ces mobilisations à géométrie variable, relayées et amplifiées par une série d'événements fortement médiatisés, le harcèlement de rue a fait l'objet d'un intérêt social et politique croissant, jusqu'à se voir récemment saisi par les politiques publiques. Des outils nouveaux ont été progressivement mis en place, à différentes échelles, visant à la fois à mieux cerner et mesurer le phénomène (innovations institutionnelles, dispositifs, enquêtes), à le prévenir (campagnes de communication, programmes pédagogiques) et à le réprimer (innovations législatives et réglementaires, formation et sensibilisation des personnels de police, etc.), notamment à partir d'inflexions ou d'innovations réglementaires et législatives, aux niveaux local et/ou national.

Certains pays européens ont ainsi mis en place des réglementations spécifiques et des mesures répressives nouvelles ont été instituées ${ }^{[2]}$. En France, c'est tout récemment que Marlène Schiappa, alors secrétaire d'État chargée de l'Égalité entre les femmes et les hommes, s'est saisie de la question et a porté la création d'un délit d'outrage sexiste.

1 L'association Stop harcèlement de rue (2014) et la filiale française de l'association américaine Hollaback (2012) sont spécifiquement consacrées à la question. L'association Osez le féminisme (2009) en a fait l'un de ses principaux chevaux de bataille.
2 Des lois de natures assez diverses permettant de pénaliser le harcèlement de rue ont été introduites notamment au niveau local à Bruxelles (Belgique), Amsterdam et Rotterdam (Pays-Bas), et au Nottinghamshire en Angleterre. Le Pérou, le Portugal et les Philippines ont introduit des législations au niveau national. Buenos Aires (Argentine) a mis en place une réglementation locale. 
Dès sa mise à l'agenda, la mesure a fait débat, y compris parmi les militant.e.s et la communauté des chercheur.e.s travaillant sur le sujet ${ }^{[3]}$. Certaines des lignes de discussion pointaient des enjeux déjà thématisés dans des phases antérieures du processus de publicisation, notamment lors de la diffusion du documentaire de Sophie Peeters, «Femme de la rue» (2012).

En effet, le film réalisé par une jeune étudiante belge (dont c'était le projet de fin d'étude) dans le quartier populaire de Molenbeek, en caméra cachée, a soulevé des réactions vives et nombreuses, après avoir documenté de façon inédite la densité et la violence des interactions subies au fil de sa mobilité piétonne, en quelques heures seulement. Or, les auteurs de ces commentaires sexistes, interpellations et sifflements, épinglés par le film, correspondaient presque tous à un type particulier (masculin, populaire, non-blanc), dont l'omniprésence était d'abord liée à la topographie même du parcours suivi par la victime-documentariste, dans un quartier marqué par l'histoire migratoire et la présence d'une pluralité de minorités culturelles ${ }^{[4]}$. Le film a été accusé de porter de façon tacite un propos raciste, en mettant en cause une minorité ethnoraciale ou ethnoculturelle. La hantise d'un ciblage de populations pauvres et issues des minorités ethniques devient le ressort d'une résistance qui, dès ce moment, se voit opposée aux modalités de politisation du problème du harcèlement de rue, jugées trop sélectives eu égard au problème général de la violence de genre, et qui conteste la façon dont les pouvoirs publics s'en saisissent.

À ce jour, la définition de politiques publiques ciblant spécifiquement le harcèlement de rue reste récente, et leur mise en œuvre est encore balbutiante. L'heure semble donc propice à un premier retour réflexif sur le phénomène, les conditions de son érection en problème public, les délimitations d'un périmètre de réponses possibles que préfigure sa définition. Quels critères et quel périmètre attribue-t-on au harcèlement de rue au moment de formuler des politiques publiques visant à y répondre? Quels comportements et quels types de victimes la définition du problème recouvre-t-elle? La reconnaissance publique de ce problème passe-t-elle nécessairement par la pénalisation? Quels en

3 La loi a été votée à l'été 2018: les comportements pouvant être qualifiés comme relevant d'un "outrage sexiste" sont depuis le $1^{\text {er }}$ août passibles d'une amende d'un montant de 90 euros minimum. Cette loi a suscité des débats dès l'annonce de sa mise en chantier à la rentrée 2017. La tribune signée par un collectif de chercheur.e.s spécialistes du genre dans le quotidien Libération en a marqué un temps fort: Brown, Elizabeth et al., 2017, "Contre la pénalisation du harcèlement de rue». Libération, 26 septembre 2017.

4 Le premier lien à faire ici est donc sans doute celui, bien documenté par les historiens et les sociologues, entre présence dans la rue (espace de sociabilité, de travail, voire de vie) et situations de pauvreté. 
sont les enjeux? Quels enseignements peut-on tirer des divers outils d'ores et déjà mis en œuvre dans d'autres pays, ou de la diversité des débats et perspectives déployés dans les différents espaces nationaux sur la question? Telles sont les principales questions auxquelles s'attachent les contributions réunies dans ce numéro spécial.

À partir d'enquêtes de terrain approfondies, il propose d'esquisser de premiers éléments de réponse à ces interrogations, relativement à la configuration politique du problème public du harcèlement de rue, à la façon dont sont envisagées et appliquées les réponses à lui apporter, en termes de prévention et de répression directes, mais aussi en ce qui concerne son impact sur les politiques de conception et de gestion des espaces publics urbains (la définition de leurs normes d'usage et conditions d'hospitalité). Ces analyses s'appuient sur des mises en perspective à la fois topographiques et chronologiques, revenant sur la genèse de la publicisation de ces questions dans une diversité de contextes nationaux, et étudiant les voies différenciées de politisation et de pénalisation auxquelles ces configurations ont donné lieu. Les mises en perspective extra-hexagonales sont ici d'autant plus utiles que le problème du harcèlement de rue a lui-même été forgé au gré de circulations internationales.

Dans cette introduction nous soulignons trois dimensions importantes dans la structuration de ces processus différenciés de politisation: 1) l'articulation complexe qui s'opère entre la diffusion internationale de notions et de paradigmes d'interprétation, apparemment homogènes, cohérents et constants, issus de milieux à la fois académiques et militants, et la diversité des configurations locales de leur réception et de leur traduction politique; 2 ) la pluralité des façons de concevoir le rapport (et le passage) entre la reconnaissance politique du problème du harcèlement de rue et les réponses publiques, notamment pénales, à lui apporter; 3 ) le caractère central qu'ont pris dans les débats auxquels a donné lieu le harcèlement de rue, la question "culturelle» et le risque de stigmatisation, en lien étroit dans les années récentes, en Europe en particulier, avec la question migratoire.

\section{Circulations internationales, configurations locales}

L'une des ambitions de cette livraison de Déviance et Société est d'étudier les voies qu'a suivies la politisation récente du harcèlement de rue dans différents contextes politiques et culturels. Les articles réunis entendent nourrir l'analyse des ressorts et des effets des multiples configurations qui se sont déployées à cette occasion, en France, aux Pays-Bas, mais aussi au-delà 
des frontières de l'Europe. Depuis l'analyse des réticences à la pénalisation des «propos publics offensants» élaborée par Laura Beth Nielsen (2000, 2004) pour les États-Unis, jusqu'à l'identification des résistances que suscite le concept même de harcèlement de rue, comme c'est le cas en Colombie, les études réunies dans ce numéro relient ces processus de publicisation à la fois à leur genèse, à leurs ancrages militant et intellectuel, et à leurs effets politiques et juridiques, notamment dans la perspective de leur pénalisation, souvent encore récente, voire embryonnaire.

La notion de "harcèlement de rue» a été forgée aux États-Unis au début des années 1980, et développée dans les travaux fondateurs de l'anthropologue Micaela di Leonardo (1981) puis de la sociologue Carol Brooks Gardner (1980, 1995). Comme le rappelle Carole Gayet-Viaud dans sa contribution, les premières recherches nord-américaines sur le sujet se sont placées à la croisée de deux champs d'analyse: d'une part, les recherches féministes portant sur les violences faites aux femmes et le harcèlement sexuel, développés notamment par la juriste féministe Catherine MacKinnon (1979), et d'autre part, les travaux de sociologie urbaine, conduisant à définir le "harcèlement public des femmes" (Gardner, 1995) comme une rupture de la norme d'«inattention civile» (Goffman, 2013) qui prévaut dans les rapports en public.

À partir des années 1990, plusieurs juristes ont commencé à plaider à la fois pour une reconnaissance publique du problème et pour la pénalisation de ces comportements (Bowman, 1993; Davis, 2002), considérant ces deux aspects comme indissociables. C'est aussi là une hypothèse défendue par L. B. Nielsen dans ses travaux (2000), dont on propose ici la traduction d'un texte devenu classique, mais peu connu du public francophone, où la sociologue du droit analyse, dans la perspective des travaux sur «la conscience du droit», les perceptions ordinaires prévalant sur le harcèlement de rue et sur l'opportunité pour le droit de s'en saisir et de le sanctionner. Dès sa genèse outre-Atlantique, la notion de harcèlement de rue s'est donc inscrite dans une perspective à la fois militante et pluridisciplinaire, à l'intersection de la sociologie, du droit et des gender studies. C'est ensuite au travers des actions de collectifs militants américains tels que Hollaback! et Stop Street Harassment que la notion a commencé de gagner graduellement une reconnaissance publique plus large. La popularisation de l'usage de la notion de harcèlement de rue date des années 2000 et 2010. L'expression est alors reprise dans le monde entier. Le phénomène était déjà étudié depuis longtemps (dès les années 1980) par des chercheuses féministes, en Europe également (par exemple les universitaires allemandes Cheryl Benard et Edit Schlaffer, 1984), mais ce n'est que deux décennies plus tard que le «harcèlement de rue» se voit consolidé en tant qu'objet de 
recherche reconnu, et qu'il devient un sujet de premier plan de militantisme et la cible d'actions publiques spécifiques, dans toute l'Europe.

Pour autant, la circulation des idées n'est jamais, rappelons-le, une simple diffusion d'idées et de répertoires. À distance des modèles «diffusionnistes essentialistes» (Chabot, Duyvendak, 2002, 698) d'étude de la circulation des idées, on porte ici attention à la façon dont l'émergence d'un débat public sur le harcèlement de rue, dans une diversité de pays, s'est faite au gré de processus de "traduction» (Latour, 1984; Callon, 1986) et d'«appropriation" (Wachtel, 1971) à chaque fois singuliers. Si la prise de conscience et la publicisation du harcèlement de rue constituent en partie des phénomènes d'envergure mondiale, leur mise à l'agenda médiatique et politique s'est faite par l'entremise d'une intégration sélective, négociée, dans des répertoires culturels nationaux (Lamont, Thévenot, 2000) différents voire divergents. La prise en compte de la diversité de ces contextes locaux éclaire la pluralité des enjeux soulevés par le phénomène et les variations possibles dans les façons de le percevoir et de l'évaluer, dans l'expérience ordinaire des citadin·e·s, comme au niveau des débats publics et des institutions, pour le juger plus ou moins tolérable, évaluer ses causes et ses effets (qu'on invoque sa dimension "pulsionnelle» ou son inscription dans des traditions culturelles), et justiciable ou non d'intervention publique, voire de répression pénale.

Ainsi que le montre l'article de Samantha Joeck, consacré aux interactions sexuées et à leur qualification dans les espaces publics d'une grande ville de Colombie, la thématisation des rapports de genre en public dans les termes du harcèlement de rue se heurte à certains obstacles dans le paysage politique colombien, obligeant les acteurs locaux à accommoder ce terme avec les schèmes de perception culturels dominants. La notion de harcèlement de rue s'y place en effet en concurrence avec la catégorie locale de "piropos" qui renvoie à une pratique socialement acceptée, voire positivement connotée. Cette catégorie et les arrière-plans normatifs qui la sous-tendent influent donc considérablement sur les possibilités locales d'appropriation de la notion de harcèlement de rue. Dans certains pays, le fait que la notion circule à une échelle internationale et qu'elle soit considérée comme «importée» peut également entrer en ligne de compte et peser sur son devenir local, l'argument pouvant être aussi bien porté au crédit de cette thématisation du problème (faisant autorité, signalant un "retard» à combler) qu'à son débit (suscitant la défiance s'il est perçu comme la menace d'un «impérialisme»).

La traduction de l'article de Laura Beth Nielsen consacré à «la conscience du droit sur le harcèlement de rue» permet elle aussi de poser certains des enjeux clés de la politisation du harcèlement de rue - en particulier la 
définition d'une intervention publique et d'une régulation juridique du phénomène - à partir d'une analyse de la perception ordinaire de ce qui est juste et injuste dans les rapports entre hommes et femmes en public (cette fois depuis la perspective états-unienne). À partir d'une enquête par entretiens, la sociologue du droit a en effet identifié des types de jugements et d'attitudes vis-à-vis de la pénalisation du harcèlement de rue qui offrent un éclairage précieux sur les tensions qu'elle soulève, et qu'on retrouve aujourd'hui toujours présentes dans maints débats sur la question.

Les trois autres contributions (originales) du numéro présentent des études de cas centrées sur la France et l'Europe. On y découvre comment, à partir de la fin des années 2000, le harcèlement de rue s'est imposé progressivement comme objet d'enquête, de mobilisation et d'action publique, dans les milieux savants, militants et au sein des institutions publiques. Les analyses proposées mettent en lumière la diversité des prises de position et des formulations politiques des enjeux relatifs au harcèlement de rue. Aux Pays-Bas, comme le montre la contribution de Mischa Dekker, dont les travaux portent sur la comparaison des situations française et néerlandaise, c'est plutôt la droite qui s'est saisie du problème dans les années 2000 et en a défini les termes, en l'inscrivant dans un périmètre où immigration, sécurité et harcèlement de rue forment un faisceau commun de questions. En France en revanche, la thématisation du phénomène a longtemps été portée exclusivement par les féministes et marquée par un fort ancrage historique à gauche. De ce fait, le couplage de la reconnaissance publique du problème avec l'élaboration de politiques pénales n'a pas reposé sur les mêmes arrièreplans ni sur les mêmes évidences dans l'un et l'autre contextes.

En France, l'extension récente du périmètre des personnes concernées (Dewey, 2010) par le problème public du harcèlement de rue, vers la droite de l'échiquier politique, s'est accompagnée de tentatives de reconfiguration des termes du problème, autour des questions de sécurité et de migration, provoquant de vives réactions chez certaines des porteuses de cause, estimant le problème instrumentalisé. La contribution de Marylène Lieber revient sur ce risque de dévoiement, en retraçant les étapes françaises de politisation de la place des femmes en ville, depuis leur invisibilisation initiale, pour montrer comment la préoccupation récente pour la transformation des villes en vue d'une meilleure hospitalité donnée aux usagères s'articule en pratique avec des opérations de gentrification, de sélection des usagères dignes d'être prises en compte et la production de «nouveaux indésirables». Ces ambivalences, voire cette relative hypocrisie des rhétoriques de l'inclusion et de l'hospitalité mobilisées, invitent à nuancer la description de la mise à l'agenda politique du problème du harcèlement de rue dans les seuls termes d'une victoire pour ses porteuses historiques. 


\section{Le devenir pénal d'une cause féministe}

Comme l'a montré Gusfield (2009) à propos de la pénalisation de la conduite en état d'ivresse, la production statistique scientifique aussi bien que la production juridique doivent être considérées comme participant à la constitution d'un problème public, plutôt que comme extérieures (et postérieures à celle-ci). Les faits scientifiques ne préexistent jamais entièrement à la définition des catégories qui permettent de les saisir et les mesurer (Cicourel, 2017). De même, le travail législatif revêt un pouvoir instituant considérable. Si ce numéro interroge l'émergence de la notion de «harcèlement de rue» et ses dynamiques générales de politisation, il porte une attention particulière à sa traduction en catégorie de l'action publique, et plus spécifiquement encore, en objet de politique pénale. Le harcèlement de rue fait désormais l'objet de lois et d'ordonnances visant à le réprimer.

En France, la loi a créé le délit d'outrage sexiste en août 2018. Les "10000" policiers de la "police du quotidien», dont les contours des missions comme l'origine des effectifs restent encore flous, devront verbaliser cette nouvelle contravention. Si les injures sexistes figuraient déjà en France dans le Code pénal ${ }^{[5]}$, au même titre que les injures discriminant les personnes en raison de leur orientation sexuelle ou de leur handicap (contravention de classe 4 passible de 750 euros d'amende), elles n'étaient cependant jusque-là, contrairement au délit d'injure raciste, jamais poursuivies par les pouvoirs publics, à moins qu'il n'y ait dépôt de plainte. Cette innovation juridique marque ainsi l'aboutissement (provisoire et contingent) d'un travail de politisation, dont le cheminement ne va jamais de soi. Cette production juridique (et pénale) est considérée par certains comme le moyen incontournable d'une reconnaissance publique véritable du problème et le passage obligé d'une lutte politique effective (c'est l'argument de Nielsen), mais peut aussi être considérée comme un dévoiement des principes et des valeurs féministes qui ont porté l'élaboration du concept et œuvré à sa diffusion.

Sur cette tension entre la dénonciation d'un problème et la formulation d'une réponse publique dans les termes d'une politique pénale

5 L'article 33 de la loi du 29 juillet 1881 (sur la liberté de la presse) définit l'injure publique: "une invective, une expression outrageante ou méprisante pouvant être entendue ou lue par un public inconnu et imprévisible, c'est-à-dire par un nombre indéterminé de personnes". 
jugée trop répressive et/ou sélective, les travaux récents consacrés au «féminisme carcéral ${ }^{[6]}$ » offrent un point de comparaison intéressant: c'est en effet un domaine où les luttes féministes ont également donné lieu à la mise en œuvre de politiques répressives, autour notamment des questions liées au viol et à la prostitution, suscitant des critiques et une réflexivité inquiète d'une partie des collectifs engagés ${ }^{[7]}$. La pénalisation du harcèlement de rue peut aussi être envisagée dans cette perspective.

La configuration du problème en termes de sécurité, de déviance et de "policing" des espaces publics urbains ne va pas non plus de soi. Elle tend à isoler le phénomène du sexisme de rue des autres espaces et formes de manifestation du sexisme (mais aussi des autres formes de trouble de la coexistence publique) et tend surtout à dramatiser la dangerosité des actes de sexisme ordinaire pour en justifier la prise en charge juridique et policière. Après leur invisibilisation et la négligence dont ces comportements ont fait l'objet pendant des décennies, la dramatisation de ces formes de déviance et d'offenses mineures passe désormais souvent par leur appariement à des formes de violence lourde, via l'idée qu'une forme d'engrenage mènerait des premières aux secondes. Comme le montre Carole Gayet-Viaud dans sa contribution, cette rhétorique, adossée à un usage discutable du concept de «continuum de violences» (Kelly, 2019), présente des similitudes frappantes avec les logiques mises au travail pour justifier, aux États-Unis puis en France, les politiques urbaines de lutte contre le crime dites de «tolérance zéro", à la fin du siècle dernier. Ce sont les mêmes thèses relatives à ce à quoi sont réputés pouvoir conduire des délits mineurs et des incivilités, qui ont justifié leur répression sévère et "précoce». L'article permet de remettre en perspective ces glissements rhétoriques survenus dans l'usage des termes de harcèlement et de continuum des violences, en revenant sur leur genèse et leurs trajectoires au fil de la politisation du problème, et en les mettant en regard des enseignements issus des politiques de lutte contre l'incivilité.

6 E. Bernstein utilise cette expression pour désigner "une formation culturelle et politique qui traduit les luttes pour la justice et la libération de la génération précédente en termes carcéraux" (Bernstein, 2012, 235, [notre traduction]).
7 Ces travaux montrent en effet comment les campagnes féministes contre les violences sexuelles ont non seulement été intégrées dans des politiques publiques carcérales, mais ont également fourni des arguments majeurs pour l'élaboration et la justification de ces politiques répressives (Bernstein, 2007; Bumiller, 2008). 


\section{Configuration culturaliste et risques de stigmatisation}

En Europe, le succès médiatique et politique de la notion de harcèlement de rue est allé de pair avec la montée en puissance des débats cristallisés autour de la dimension culturelle du problème. Ainsi que l'ont montré plusieurs travaux, à l'instar de ceux de Jasbir Puar (2007) sur l'«homonationalisme», les combats pour les droits des femmes et des personnes LGBTQI+ (Lesbian Gay Bi Trans Queer Intersexual and others) s'entrelacent désormais fréquemment avec le développement de politiques nationalistes et répressives (Fassin, 2010; Farris, 2017). Dans les débats publics, le problème des violences faites aux femmes est de plus en plus corrélé aux populations issues de l'immigration (Roggeband, Verloo, 2007). Ce sont souvent des quartiers à forte mixité culturelle et ethnique qui ont été mis au centre des débats médiatiques et qui ont accéléré la politisation et la mise à l'agenda public de ces questions. Lors de ces débats, la «culture ${ }^{[8]}$ » des hommes issus de l'immigration s'est vue mobilisée pour expliquer leurs comportements sexistes. Ces cadrages du problème de harcèlement de rue concourent à produire une «culturalisation» du problème ${ }^{[9]}$ (Duyvendak et al., 2016) qui, bien que suscitant de vives résistances, tend à s'installer durablement, à la faveur de faits divers au fort retentissement médiatique, à l'échelle européenne.

Un tournant en la matière a sans doute été la série d'agressions sexuelles ayant pris place la veille du Nouvel An 2016 à Cologne. Associant, dans un fait divers glaçant, des regroupements d'hommes migrants et des faits multiples de violences sexuelles, l'événement a précipité la «droitisation» de la question. En France, un an après ces événements de Cologne, la controverse a été relancée par une pétition émise par des habitants du quartier La Chapelle-Pajol à Paris, relayée par le journal Le Parisien qui titrait «Les femmes, une espèce en voie de disparition au cœur de Paris » ${ }^{[10]}$, donnant l'occasion à des figures politiques d'envergure nationale de droite, voire d'extrême droite, de se positionner sur un sujet (et d'afficher un «féminisme») jusque-là peu investi.

Si la droite néerlandaise a systématiquement présenté le problème comme essentiellement créé par des jeunes issus de l'immigration

8 Pour une critique systématique de ces usages réifiants et homogénéisants du concept de culture, qui creusent l'altérité de celles et ceux ainsi désignés, voir AbuLughod, 2010.

9 On entend par là les processus par lesquels des acteurs en viennent à concevoir un problème comme principalement lié à la culture de certains groupes sociaux.

10 Change.org., 2017, La Chapelle \& Pajol: Les femmes, espèce en voie de disparition au cœur de Paris: https://www. change.org/p/emmanuel-macron-lesfemmes-esp \%C3\%A8ce-en-voie-dedisparition-au-coeur-de-paris. 
et a réussi à imposer ce cadrage dans les médias, en France, la perspective d'une pénalisation a heurté une partie des porteur.se.s de cause du harcèlement de rue, fortement ancré·e·s à gauche. Des voix s'élèvent pour dénoncer les potentiels effets stigmatisants des politiques publiques à l'œuvre ou en gestation, vis-à-vis des hommes issus des classes populaires et/ou de l'immigration. Elles s'inquiètent de la mise en concurrence de deux dimensions également importantes de leur engagement: l'émancipation des femmes d'une part, et le rejet de toute stigmatisation des minorités d'autre part (Hancock, Lieber 2017; Delphy, 2006). Les politiques publiques mises en œuvre au nom de ce combat suscitent ainsi des réserves et parfois même des oppositions franches, y compris chez ceux et celles-là mêmes qui les avaient appelées de leurs vœux. Plusieurs contributions de ce numéro reviennent sur ces enjeux et leur rôle déterminant dans la structuration des débats.

\section{Présentation des contributions}

La contribution de Mischa Dekker retrace les processus différenciés de pénalisation du harcèlement de rue en France et aux Pays-Bas. Si on parle souvent d'une prise de conscience mondiale de la question du harcèlement, l'enquête ethnographique conduite dans les deux pays montre que la définition du problème et du phénomène du harcèlement de rue même est fortement ancrée dans des contextes locaux. Si en France des politicien.ne.s de gauche et des militant·e.s féministes ont été les premier-e.s entrepreneur.se.s de cause du phénomène, cadrant le harcèlement de rue comme un problème de violences faites aux femmes et de domination masculine, aux Pays-Bas, ce sont des représentants politiques de la droite qui sont les premiers, à partir de 2009, à mettre la question à l'agenda politique, présentant le harcèlement plutôt comme une menace à l'ordre public, lié à l'immigration. L'auteur retrace comment, malgré ces différences, les législateurs travaillant sur la mise en place de lois sur le harcèlement de rue font face à une critique similaire, exprimée par des féministes et des politicien.ne.s de gauche: le risque de pénaliser disproportionnellement les hommes issus des classes populaires et de l'immigration. L'article analyse la manière dont la "hantise» de stigmatiser influe sur la mise en place des lois pénales sur le harcèlement de rue, en traçant comment certains acteurs arrivent à concevoir une loi comme étant «raciste» ou "sélective», et la façon dont les législateurs de ces deux pays négocient cette critique.

Dans son article, Carole Gayet-Viaud propose de revenir sur la constitution du harcèlement de rue comme problème public en France, à 
partir d'une analyse critique de l'usage des notions de harcèlement et de continuum de violences auquel cette politisation s'est adossée. Si la thématisation des rapports de genre en public dans les termes du harcèlement de rue a permis la saisie et la dramatisation d'un ensemble hétérogène de gestes et de paroles, initialement conçus comme négligeables et anecdotiques, qui se sont avérés décisifs dans la politisation du phénomène, l'autrice montre que le couplage de la notion de harcèlement, avec la notion de continuum de violences, a des conséquences problématiques au moment de traduire cette reconnaissance publique en politique pénale. L'analyse souligne la proximité des rhétoriques de «l'engrenage» ainsi produites avec les théories mobilisées, au cours des décennies 1990 et 2000, pour justifier le développement de politiques pénales visant les incivilités (lesquelles avaient alors exclu et ignoré, sans devoir s'en justifier, les phénomènes de harcèlement sexiste). L'article invite à envisager les limites d'une approche du harcèlement de rue adossée à la notion de continuum des violences, dès lors qu'elle s'applique à des individus singuliers, et non à une culture collective.

Les conséquences politiques de la lutte contre le harcèlement de rue sont également au cœur de la contribution de Marylène Lieber. Elle montre que les politiques publiques d'aménagement des villes nourrissent des processus de gentrification qui sont pour le moins ambivalents, eu égard à leurs objectifs affichés. Elles tendent, en effet, à infléchir la conception des espaces publics urbains dans le sens d'une moindre hospitalité envers les populations les plus défavorisées, par la production, au nom de la sécurité des femmes, de nouvelles catégories d'indésirables. La mise en place de ces politiques publiques s'inscrit en effet, note l'autrice, dans un "paradigme des espaces publics comme vitrines des villes» qui mène à l'exclusion de certaines catégories d'hommes de ces espaces (spécifiquement, les hommes appartenant aux minorités ethniques visibles) ce qui tend à renforcer des processus de gentrification déjà à l'œuvre, et à nourrir la confusion entre la "qualité» des espaces publics urbains et leur homogénéité sociale et ethno-raciale ${ }^{[11]}$. Ce constat d'une «spatialisation» et d'une «racialisation» des politiques antisexistes invite alors à repenser les présupposés qui guident la lutte contre le harcèlement de rue, ainsi que le qualitatif «de rue» dans l'expression elle-même.

La contribution de Samantha Joeck montre quant à elle le travail de «traduction» nécessaire pour acclimater la notion de harcèlement de rue, pour en affirmer la pertinence comme catégorie descriptive et

11 Cet argument, mis en avant dans la tribune de Libération mentionnée précédemment, est également avancé par l'association féministe musulmane Lallab (2017). 
en faire un objet pris au sérieux dans le débat public colombien, aux échelles locale et nationale. La conceptualisation des rapports hommesfemmes en public dans les termes du harcèlement de rue s'y heurte, en effet, à la prévalence du terme de "piropos», et aux connotations positives auxquelles la pratique est, par son entremise, couramment associée. L'analyse d'entretiens relatifs à l'expérience ordinaire que font les femmes de différents milieux sociaux de ces interactions genrées en public, permet d'éclairer la multiplicité des facteurs qui influencent la perception de ce qui se passe et la définition de la situation (Thomas, 1969) qui prévaut dans ces contextes - le caractère sympathique, comique, ou offensant, voire menaçant, de telle interpellation verbale, ou de tel regard - et donc d'interpréter les conditions de réception, d'interprétation, et finalement d'acceptabilité sociale des conduites ainsi désignées.

On retrouve, dans ces distinctions et ces potentielles disputes interprétatives, un écho de tensions et controverses récurrentes en anthropologie notamment, entre féminisme et culturalisme (Moller Okin, 1999; Abu-Lughod, 2010; Gayet-Viaud, 2010), opposant le respect de la pluralité des traditions culturelles et les exigences d'un universalisme égalitaire; mais aussi de tensions relatives à la définition des frontières entre pratiques de séduction et gestes de prédation ${ }^{[12]}$ voire d'agression (le terme de "harcèlement» visant précisément à requalifier une partie des pratiques susceptibles d'être désignées comme des «compliments» ou des "plaisanteries» en offenses et en gestes d'agression). On pense notamment aux débats ayant pris place en France autour de «l'affaire DSK» d'abord, relancés sous des formes voisines avec le mouvement \#MeToo (et \#BalanceTonPorc) et qui ont opposé, dans des termes souvent très caricaturaux, les caractéristiques réelles ou supposées d'une séduction «à la française» qu'il semblait nécessaire de défendre contre les attaques du "puritanisme américain", au nom d'un patrimoine culturel hexagonal menacé, ou l'exaltation inquiète d'un «droit d'importuner ${ }^{[13]}$ » qui a fait également controverse.

12 L'impossibilité de définir une frontière entre formes acceptables et formes agressives d'interpellation masculine des femmes a été considérée, en France notamment, comme symptomatique d'une euphémisation du problème. La question peut être abordée différemment: on trouve dans les travaux de chercheuses féministes un questionnement tout à fait sérieux sur les apparentements possibles entre les formes de séduction, la distribution genrée ordinaire des rôles sexuels, et l'existence voire la légitimité (relative) de certaines pratiques et formes d'agression et de violences faites aux femmes, que ce soit par l'entremise d'une opposition actif/passive, ou par "l'accaparement masculin de l'initiative" (Gardner, 1995), par exemple.

13 Une tribune portant ce titre, initiée par l'écrivaine $\mathrm{S}$. Chiche et co-signée par de nombreuses personnalités du monde de la culture, dont Catherine Deneuve, est parue dans Le Monde le 9 janvier 2018. 
Les trajectoires et les espaces de production normative, de définition des catégories et de qualification des pratiques se voient donc éclairées par ces perspectives plurielles sur leur mise à l'épreuve située: les contributions donnent à voir les processus de formation du jugement et de mise en discussion des idées, dans les expériences personnelles ordinaires, dans les controverses politiques et médiatiques, dans les discussions académiques et savantes, et dans les espaces militants et pédagogiques. Les circulations entre ces espaces sont nombreuses et moins unilatérales qu'on ne l'imagine parfois. C'est leur considération conjointe qui permet d'affiner la compréhension du problème public du «harcèlement de rue» tel qu'il s'est aujourd'hui configuré dans une diversité d'espaces nationaux.

Ce numéro propose enfin la traduction française (par Cyril Le Roy ${ }^{[14]}$ ) d'un texte classique de Laura Beth Nielsen, Professeure de sociologie du droit à la Northwestern University. Ses travaux, qui s'inscrivent dans la perspective des «Legal Consciousness Studies» (Ewick, Silbey, 1998), constituent un jalon important des réflexions qui se sont développées sur le sujet au cours des dernières décennies, et qu'il semble par conséquent utile de rendre disponible au lectorat francophone. Les travaux de L. B. Nielsen (notamment son ouvrage de 2004 License to Harass) analysent la façon dont le phénomène est perçu, la manière dont les personnes ordinaires se figurent la pertinence des réponses qui pourraient ou devraient lui être apportées, en termes de légitimité et d'efficacité, notamment s'agissant d'une éventuelle pénalisation. Dans «Situer la conscience du droit: expériences et attitudes des citoyens ordinaires face au droit sur le harcèlement de rue», Nielsen fournit ainsi une analyse des principales tensions générées par l'idée de pénaliser le harcèlement de rue. Dans sa perspective, ces difficultés sont des obstacles qu'il s'agit de lever. Mais son analyse reste pertinente que l'on partage ou non ce point de vue. Elle y étudie en effet finement les raisonnements politiques et moraux qui sous-tendent les opinions relatives à la pénalisation. Ce faisant, elle donne à voir plusieurs lignes de clivage et de controverse évoquées précédemment, éclairant leur façon de structurer les prises de position.

Les personnes interrogées par Nielsen dans son enquête se prononcent majoritairement contre la judiciarisation des offenses publiques, mais divers motifs justifient cette opposition. Les mots utilisés et les raisonnements mobilisés par les enquêté·e·s permettent à l'autrice d'identifier quatre paradigmes distincts qui mettent l'accent respectivement sur la liberté d'expression, l'autonomie, l'impraticabilité et la défiance vis-à-vis des autorités. Le premier motif de réticence à la pénalisation 
tient à ce que Nielsen appelle le "paradigme de l'autonomie». C'est l'idée que le recours au droit suscite, chez certaines personnes, le sentiment d'être réduites au statut de victimes passives et se voient nier la capacité de se défendre elles-mêmes, ce qui les menace dans leur autonomie ${ }^{[15]}$. Nielsen pointe également l'importance du «paradigme de la méfiance de l'autorité»: pour ses enquêté.e.s, la peur du profilage ethnique constitue l'une des sources principales de scepticisme eu égard à la pénalisation, $a$ fortiori chez les enquêtés masculins afro-américains (56\%).

Or, ces deux inquiétudes irriguent toujours les débats contemporains sur le harcèlement de rue. Si la constitution du harcèlement de rue en problème public vise à garantir à tou.te-s et aux femmes notamment, un usage autonome et libre de l'espace public - en protégeant les personnes contre la banalisation des violences quotidiennes qu'elles y subissent - elle suscite à son tour la crainte de constituer une atteinte à l'autonomie des personnes: en réduisant les femmes et les personnes LGBTQI+ au statut de victimes passives, ou en contribuant à la stigmatisation d'hommes issus de minorités (voir notamment les contributions de Marylène Lieber et de Mischa Dekker). La contribution de L. B. Nielsen à la réflexion sur la pénalisation du harcèlement de rue garde donc aujourd'hui encore toute sa pertinence. Elle nous semble nourrir un dialogue d'autant plus fécond avec les articles réunis dans ce numéro ${ }^{[16]}$ que ceux-ci invitent, chacun à leur manière, à prolonger par l'enquête empirique, l'analyse des raisonnements pratiques et des tensions à l'œuvre dans la perception du harcèlement de rue et dans l'élaboration des réponses publiques à lui apporter, en suivant leurs développements empiriques variés dans une diversité de contextes nationaux, leurs traductions locales et leurs manières de transformer «un malaise vécu en injustice» (Boussaguet, 2009, 228).

\section{Carole Gayet-Viaud}

CNRS-CESDIP (UVSQ-UCP-ministère

de la Justice) et EHESS-CEMS CESDIP

43, boulevard Vauban - 78280 Guyancourt

carole.gayetviaud@cesdip.fr

\section{Mischa Dekker}

Doctorant en co-tutelle entre I'EHESS-LIER

et I'Université d'Amsterdam-Political Sociology

EHESS-LIER

10, rue Monsieur le Prince -75006 Paris

mischa.dekker@sciencespo.fr

$15 \mathrm{Y}$. Barthe (2017) affirme que cette tension entre "victimisation» et "responsabilisation" structure nombre de problèmes publics.
16 Nous remercions ici l'ensemble des relecteurs et relectrices anonymes des articles, ainsi que l'éditrice de la revue Valérie Martin, pour leur travail et leur accompagnement précieux dans la confection de ce numéro. 
ABU-LUGHOD L., 2010 [1991], Écrire contre la culture. Réflexions à partir d'une anthropologie de l'entre-deux, in CEFAÏ D. (dir.), L'engagement ethnographique, Paris, Éditions de l'EHESS, coll. "Temps et lieux".

BARTHE Y., 2017, Les retombées du passé, Le paradoxe de la victime, Paris, Seuil.

BENARD C., SCHLAFFER E., 1984,

The Man in the Street: Why He Harasses, in VERTA T.A., WHITTER N., RICHARDSON L. (Eds.), Feminist frontiers IV, New York, N.Y., McGraw-Hill.

BERNSTEIN E., 2007, The Sexual Politics of the "New Abolitionism", Differences, $18,5,128-151$.

BERNSTEIN E., 2012, Carceral Politics as Gender Justice? The Traffic in Women and Neoliberal Circuits of Crime, Sex, and Rights, Theory and Society, 41, 233-259.

BOWMAN C.B., 1993, Street Harassment and the Informal Ghettoization of Women Cornell Law Faculty Publications, 106, 3, 517-580.

BOUSSAGUET L., 2009, Les "faiseuses" d'agenda. Les militantes féministes et l'émergence des abus sexuels sur mineurs en Europe, Revue française de science politique, 59, 2, 221-246.

BUMILLER K., 2008, In an Abusive State: How Neoliberalism Appropriated the Feminist Movement Against Sexual Violence, Durham, NC, Duke University Press.

CALLON M., 1986, Éléments pour une sociologie de la traduction. La domestication des coquilles SaintJacques dans la Baie de Saint-Brieuc, L'Année sociologique, 36, 169-208.

CEFAÏ D., TERZI C. (dir.), 2012, L'expérience des problèmes publics, Raisons pratiques, 22, Éditions de I'EHESS.
CHABOT S., DUYVENDAK J.W., 2002, Globalization and transnational diffusion between social movements: Reconceptualizing the dissemination of the Gandhian repertoire and the "coming out" routine, Theory and Society, 31, 6, 697-740.

CICOUREL A., 2017 [1968] La justice des mineurs au quotidien de ses services, Genève, IES/HETS.

DAVIS D.E., 1994, The Harm that has no Name: Street Harassment, Embodiment, and African American Women, UCLA Women's Law Journal, 4, 2, 133-178.

DELPHY C., 2006, Antisexisme ou antiracisme? Un faux dilemme, Nouvelles Questions Féministes, 25, 1, 59-83.

DEWEY J., 2010 [1927], Le public et ses problèmes, Paris, Gallimard.

DI LEONARDO M., 1981, Political Economy of Street Harassment, Aegis. Magazine on Ending Violence Against Women, 51-57.

DUYVENDAK J.W., GESCHIERE P., TONKENS E. (Eds.), 2016, The Culturalization of Citizenship. Belonging \& Polarization in a Globalizing World, London, Palgrave/Macmillan.

EWICK P., SILBEY S., 1998, The Common Place of Law: Stories from Everyday Life, Chicago, University of Chicago Press.

FARRIS S.R., 2017, In the Name of Women's Rights. The Rise of Femonationalism, Durham, Duke University Press.

FASSIN É., 2010, National Identities and Transnational Intimacies: Sexual Democracy and the Politics of Immigration in Europe, Public Culture, 22, $3,507-529$.

GARDNER C.B., 1980, Passing By: Street Remarks, Address Rights, and the Urban Female, Sociological Inquiry, 50, 3-4, 328356. 
GARDNER C.B., 1995, Passing By: Gender and Public Harassment, Los Angeles, University of California Press.

GAYET-VIAUD C., 2010, La critique du concept de culture. Ethnographies féministes et subalternes, in CEFAÏ D. (dir.), L'engagement ethnographique, Éditions de l'EHESS, coll. "Temps et lieux".

GAYET-VIAUD C., 2014, Le lien civil en crise?, Bruxelles, Éditions Yapaka [réédition 2015, Éditions Fabert].

GOFFMAN E., 2013 [1968], Comment se conduire dans les lieux publics, Paris, Economica.

GUSFIELD J., 2009, La culture des problèmes publics. L'alcool au volant: la production d'un ordre symbolique, Economica, coll. "Études Sociologiques».

HANCOCK C., LIEBER M., 2017, Refuser le faux dilemme entre anti-sexisme et antiracisme. Penser la ville inclusive, Annales de la recherche urbaine, "Le genre urbain ", 112, 16-25.

KEARL H., 2010, Stop Street Harassment. Making Public Spaces Safe and Welcoming for Women, Santa Barbara, $\mathrm{CA}$, Praeger.

KELLY L., 2019 [1987], Le continuum de la violence sexuelle, Cahiers du Genre, 66, 1, 17-36.

LALLAB, 2017, 8 raisons de s'opposer à la pénalisation du harcèlement de rue, [en ligne] http://www.lallab.org/8raisons-de-sopposer-a-la-penalisation-duharcelement-de-rue/.

LAMONT M., THÉVENOT L. (Eds.), 2000, Rethinking Comparative Cultural Sociology Cambridge, Cambridge University Press.

LATOUR B., 1984, Les Microbes. Guerre et paix, suivi de Irréductions, Paris, Métailié, coll. "Pandore».
LIEBER M., 2008, Genre, violences et espaces publics. La vulnérabilité des femmes en question, Paris, Presses de Sciences Po.

MACKINNON C., 1979, The Sexual Harassment of Working Women: A Case of Sex Discrimination, New Haven, Yale University Press.

MOLLER OKIN S., 1999, Is Multiculturalism Bad For Women?, in COHEN J., HOWARD, M. (Eds.), Is Multiculturalism Bad for Women? Princeton, Princeton University Press.

NIELSEN L.B., 2000, Situating Legal Consciousness: Experiences and Attitudes of Ordinary Citizens about Law and Street Harassment, Law \& Society Review, 34, 4, 1055-1090.

NIELSEN L.B., 2004, License to Harass: Law, Hierarchy, and Offensive Public Speech, Princeton, N.J., Princeton University Press.

PEETERS S., 2012, Femme de la rue, Travail de fin d'étude à la Haute école Rits [film documentaire].

PUAR J.K., 2007, Terrorist Assemblages: Homonationalism in Queer Times, Durham, London, Duke University Press.

ROGGEBAND C., VERLOO M., 2007,

Dutch Women are Liberated, Migrant Women are a Problem: The Evolution of Policy Frames on Gender and Migration in the Netherlands, 1995-2005, Social Policy and Administration, 41, 3, 271-288.

THOMAS W., 1969 [1923], The unadjusted girl, Montclair, N.J., Patterson Smith,

TISSOT S., 2007, L'État et les quartiers, genèse d'une catégorie de l'action publique, Paris, Seuil, coll. "Liber».

WACHTEL N., 1971, La Vision des Vaincus - Les Indiens du Pérou devant la Conquête Espagnole (1530-1570), Paris, Gallimard. 